\title{
UTILIZAÇÃO DA FOSFINA EM COMBINAÇÃO COM O DIÓXIDO DE CARBONO NO CONTROLE DO RHYZOPERTHA DOMINICA (F.) ${ }^{1}$
}

\author{
ANA PAULAMARTINAZZO², LÊDA RITA D'ANTONINO FARONI ${ }^{3}$, \\ PEDRO AMORIMBERBERT ${ }^{4}$ e FERNANDO PINHEIRO REIS ${ }^{5}$
}

\begin{abstract}
RESUMO - Objetivou-se avaliar o efeito da utilização de fosfina, associada a uma atmosfera rica em dióxido de carbono $\left(\mathrm{CO}_{2}\right)$, no controle efetivo do inseto Rhyzopertha dominica (F.) em todas as suas fases de desenvolvimento. Para isso, foram realizadas fumigações com atmosfera sintética contendo $21 \%$ de $\mathrm{CO}_{2}$ e $79 \%$ de $\mathrm{N}_{2}$ associada a níveis reduzidos de fosfina $\left(0,25,0,50\right.$ e $\left.0,75 \mathrm{~g} \mathrm{~m}^{-3}\right)$. Para posterior comparação dos resultados, foram realizados dois tratamentos com ar ambiente associado às dosagens de zero e $1,00 \mathrm{~g} \mathrm{~m}^{-3}$ de fosfina. Todos os tratamentos foram realizados em três períodos de exposição $\left(24,72\right.$ e 120 horas), à temperatura de $29^{\circ} \mathrm{C}$ e umidade relativa de $60 \%$. A análise dos resultados permitiu concluir que o aumento no período de exposição resultou no aumento da eficácia dos tratamentos em atmosferas sintética e ambiente. O controle efetivo de todas as fases do inseto foi obtido, em geral, com 0,50 e $0,75 \mathrm{~g} \mathrm{~m}^{-3}$ de fosfina $+\mathrm{CO}_{2}$, em período de exposição de 120 horas. Ovos e adultos foram as fases mais suscetíveis, e pupa, a mais resistente.
\end{abstract}

Termos para indexação: controle de insetos, métodos de controle de pragas, armazenamento em atmosfera controlada, grãos.

\section{COMBINATION OF PHOSPHINE AND CARBON DIOXIDE ON THE CONTROL OF RHYZOPERTHA DOMINICA (F.)}

\begin{abstract}
The objective of this research was to evaluate the effect of low levels of phosphine associated with atmospheres containing high levels of carbon dioxide $\left(\mathrm{CO}_{2}\right)$ on the effective control of all life stages of the insect Rhyzopertha dominica (F.). To this purpose, fumigations were carried out using a synthetic atmosphere containing $21 \%$ of $\mathrm{CO}_{2}$ and $79 \%$ of $\mathrm{N}_{2}$ associated with the following levels of phosphine: $0.25,0.50$, and $0.75 \mathrm{~g} \mathrm{~m}^{-3}$. Results were compared with mortality rates achieved with ambient air containing zero and $1.00 \mathrm{~g} \mathrm{~m}^{-3}$ of phosphine. All tests were carried out using three levels of exposure time (24, 72, and 120 hours), and one level of temperature and relative humidity, $29^{\circ} \mathrm{C}$ and $60 \%$, respectively. Analyses of the results indicated that increasing the exposure time resulted in an increase in mortality rates in all tests conducted with synthetic and ambient air. Effective control of all life stages of $R$. dominica was only achieved with atmospheres containing $21 \%$ of $\mathrm{CO}_{2}$ associated with phosphine concentration levels of 0.50 and $0.75 \mathrm{~g} \mathrm{~m}^{-3}$ in 120 hours exposure time. In general, eggs and adults were the most susceptible insect stages, and pupae the most resistant.
\end{abstract}

Index terms: insect control, pest control methods, controlled atmosphere storage, grain

\footnotetext{
${ }^{1}$ Aceito para publicação em 15 de julho de 1999. Extraído da dissertação de mestrado, apresentada pelo primeiro autor à Universidade Federal de Viçosa (UFV), Viçosa, MG. Financiado pela FAPEMIG e White Martins Gases Industriais S.A.

${ }^{2}$ Eng. Agríc, M.Sc., Dep. de Engenharia Agrícola, UFV, CEP 36571-000 Viçosa, MG

${ }^{3}$ Eng. Agrôn., D.Sc., Prof. Adjunto, Dep. de Engenharia Agrícola, UFV. E-mail: 1faroni@mail.ufv.br

${ }^{4}$ Eng. Agríc., Ph.D., Dep. de Engenharia Agrícola, UFV. E-mail: pberbert@mail.ufv.br

${ }^{5}$ Eng. Agrôn., M.Sc., Prof. Adjunto, Dep. de Informática, UFV. E-mail: fpreis@dpi.ufv.br
}

\section{INTRODUÇÃO}

O inseto Rhyzopertha dominica (F.), Coleoptera, Bostrichidae, descrito primeiramente por Fabricius em 1792, é uma das pragas mais destrutivas dos grãos armazenados em todo o mundo. É considerado de grande nocividade no armazenamento do trigo e arroz beneficiado ou com casca, ocorrendo também em sorgo, milho, cevada e centeio (Gallo et al., 1988). O controle químico, principalmente na forma de fumigação, tem sido uma prática corriqueira na desinfestação de grãos armazenados infestados por inse- 
tos-praga como o $R$. dominica, destacando-se a fosfina como o fumigante mais utilizado atualmente. Relatos sobre o surgimento de mecanismos de resistência a tratamentos químicos em várias espécies de insetos têm sido cada vez mais constantes. No Brasil, ainda são poucas as informações disponíveis sobre a resistência de insetos a inseticidas, principalmente à fosfina. Trabalhos realizados por Pacheco et al. (1990) e Sartori et al. (1990) constataram a resistência à fosfina em populações de S. oryzae, $R$. dominica, Cryptolestes spp. e T. castaneum, provenientes de diversos estados brasileiros. Price \& Mills (1988) observaram que, tanto para grupos resistentes quanto para os suscetíveis, o tempo de exposição à fosfina é um fator mais crítico que a dosagem. Contudo, para o controle de populações resistentes, é necessário expor os insetos a dosagens elevadas por longos períodos.

Além da resistência aos agentes químicos, a possibilidade de intoxicação dos operadores e a presença de resíduos nos alimentos levaram à busca de alternativas menos danosas ao homem e que proporcionassem menor impacto ambiental. Uma dessas alternativas é a proteção do produto armazenado por meio da redução da concentração de $\mathrm{O}_{2}$ presente nos espaços intergranulares, com a introdução de um gás rico em $\mathrm{CO}_{2}$ em silos e armazéns comerciais. Pesquisas recentes têm demonstrado que são necessárias dosagens elevadas de dióxido de carbono para o controle efetivo de pragas (Leong \& Ho, 1994; Gonçalves, 1997). A utilização da associação de dióxido de carbono com fosfina apresenta-se como uma alternativa para reduzir tanto a concentração normalmente necessária de ambos os gases quanto o tempo de exposição necessário para o controle efetivo dos insetos. De acordo com Mueller (1994), a associação de atmosferas ricas em dióxido de carbono com fosfina, em temperaturas elevadas, provoca um grande estresse nos insetos, permitindo, assim, que baixas concentrações de fosfina sejam mais eficientes em um menor período de exposição que a dose normalmente recomendada. São escassos os dados na literatura sobre a composição ideal da atmosfera modificada em mistura com outro fumigante para o controle ou a eliminação de pragas de grãos armazenados em condições tropicais.
Este trabalho teve como objetivo avaliar o efeito de doses reduzidas de fosfina, associadas a uma atmosfera rica em dióxido de carbono, no controle de todas as fases de desenvolvimento do inseto-praga R. dominica.

\section{MATERIAL E MÉTODOS}

O trabalho foi realizado no Setor de Pré-Processamento de Produtos Agrícolas do Departamento de Engenharia Agrícola, Universidade Federal de Viçosa, Viçosa, MG. Para avaliação do efeito de atmosferas modificadas, associadas a níveis reduzidos de fosfina, no controle de pragas de grãos armazenados utilizaram-se grãos de trigo (Triticum aestivum L.) infestado com $R$. dominica.

Para a obtenção de $R$. dominica em todos os seus estádios de desenvolvimento, realizou-se uma criação contínua em câmara climática à temperatura de $32 \pm 2^{\circ} \mathrm{C}$ e umidade relativa de $60 \pm 5 \%$, que correspondem às condições ótimas para o desenvolvimento dessa espécie. A dieta para toda a criação foi constituída de trigo semitriturado, não-tratado, com teor de umidade em torno de $13 \%$ b.u..

A dose mínima de fosfina recomendada pelos fabricantes para o controle efetivo de insetos em grãos armazenados é de $1,00 \mathrm{~g} \mathrm{~m}^{-3}$ (Compêndio de defensivos agrícolas, 1996). Sendo assim, o experimento foi realizado utilizando-se três dosagens reduzidas de fosfina $(0,25,0,50$ e $\left.0,75 \mathrm{~g} \mathrm{~m}^{-3}\right)$, associadas a uma atmosfera sintética contendo $79 \%$ de $\mathrm{N}_{2}, 21 \%$ de $\mathrm{CO}_{2}$ e $0 \%$ de $\mathrm{O}_{2}$, em volume.

Para a comparação dos resultados, os insetos foram submetidos a dois tratamentos com ar atmosférico (79\% de $\mathrm{N}_{2}, 0,03 \%$ de $\mathrm{CO}_{2}$ e $21 \%$ de $\mathrm{O}_{2}$ ), utilizando-se as dosagens de zero e $1,00 \mathrm{~g} \mathrm{~m}^{-3}$ de fosfina. A fosfina foi obtida a partir da reação do fosfeto de alumínio (AlP), em forma de pastilhas, com a água contida no ar atmosférico.

Foram analisados três períodos de exposição $(24,72$ e 120 horas) e apenas um nível de temperatura e umidade relativa, $29^{\circ} \mathrm{C}$ e $60 \%$, respectivamente. $\mathrm{O}$ delineamento experimental foi inteiramente casualizado, composto de 13 tratamentos com três repetições para cada tratamento, num total de 39 parcelas. Esse procedimento visava a identificação das condições ideais de controle dos insetos com a atmosfera modificada, tomando como base o controle obtido com a dose recomendada $\left(1,00 \mathrm{~g} \mathrm{~m}^{-3}\right) \mathrm{em}$ ar ambiente.

Para a contenção dos grãos de trigo infestados com insetos foram construídos três cilindros de $0,15 \mathrm{~m}$ de diâmetro e $0,20 \mathrm{~m}$ de altura em chapa galvanizada. O fundo dos cilindros foram construídos com tela metálica, para permitir melhor difusão dos gases, com a parte superior aberta. Inicialmente, colocava-se $1,5 \mathrm{~kg}$ de trigo não-infes- 
tado em cada um dos cilindros. A seguir, amostras de trigo contendo insetos da espécie $R$. dominica em todas as suas fases de desenvolvimento eram distribuídas em pequenos copos de plástico, cobertos por um tecido permeável de malha fina, porém resistente o suficiente para impedir a saída dos insetos adultos.

As fumigações foram realizadas em uma câmara com paredes metálicas com volume útil de $1,3 \mathrm{~m}^{3}$ e que continha um dispositivo para controle da temperatura, constituído de uma resistência elétrica de $100 \mathrm{~W}$, conectada a um termostato com faixa de trabalho entre $10^{\circ} \mathrm{C}$ e $60^{\circ} \mathrm{C}$ e precisão de $\pm 1^{\circ} \mathrm{C}$. Além desse sistema para controle de temperatura, instalou-se no interior da câmara um conjunto de termopares tipo T para medição das temperaturas de bulbo seco e bulbo úmido.

Para a criação e manutenção da atmosfera sintética, um sistema para injeção e exaustão de gases foi acoplado à câmara. Este sistema era constituído por um cilindro contendo gás, válvulas, conexões e tubulações. A câmara continha um fundo falso em chapa perfurada para apoio dos recipientes contendo grãos infestados com insetos e possuía uma abertura lateral de seção quadrática de $0,35 \mathrm{~m}$ de lado.

O procedimento inicial consistia no acionamento do dispositivo para o controle da temperatura da câmara Assim que a temperatura se estabilizava em torno de $29^{\circ} \mathrm{C}$, os cilindros contendo as amostras infestadas com $R$. dominica eram colocados sobre o fundo da câmara. A seguir, pesava-se a quantidade necessária de fosfina em balança com precisão de $0,01 \mathrm{~g}$ e o produto era colocado no interior da câmara junto aos cilindros contendo as amostras. A abertura lateral de acesso era então fechada, utilizando-se uma tampa com juntas de borracha e parafusos, e a vedação era feita com um produto à base de silicone Com as válvulas de admissão e exaustão abertas, iniciavase o processo de injeção do gás sintético. Como não foi possível fabricar gás sintético contendo a quantidade de água necessária para produzir uma atmosfera com a umidade relativa desejada (60\%), foi necessário construir um dispositivo para umidificação do gás in loco. O gás umidificado penetrava na câmara pela parte lateral inferior, expulsando gradualmente o ar ambiente. As concentrações dos componentes do gás de exaustão $\left(\mathrm{O}_{2}\right.$ e $\left.\mathrm{CO}_{2}\right)$ eram continuamente monitoradas, e quando se verificava que o porcentual desses gases havia atingido $0 \%$ e $21 \%$, respectivamente, a válvula de exaustão era imediatamente fechada. Para o monitoramento da concentração dos componentes da atmosfera sintética, utilizou-se um analisador de gases modelo 7800P, fabricado pela Nova Analytical Systems.

As temperaturas de bulbo seco e úmido no interior da câmara, além da temperatura ambiente, eram medidas e registradas automaticamente a cada intervalo de 5 minutos, utilizando-se um sistema de aquisição de dados modelo Hydra 2625A, fabricado pela Fluke Corporation.

Para avaliação do efeito da fumigação sobre os insetos, após o término de cada tratamento, as amostras eram retiradas e mantidas em câmaras climáticas com temperatura de $32 \pm 2{ }^{\circ} \mathrm{C}$ e $60 \pm 5 \%$ de umidade relativa, condições consideradas ótimas para o seu desenvolvimento. Depois de 48 horas, contava-se o número de insetos adultos sobreviventes. Para os demais estádios, as avaliações eram feitas de cinco em cinco dias até que ovos, larvas e pupas tivessem tempo suficiente para atingir a forma adulta. A mortalidade encontrada foi corrigida através da fórmula de Abbot, para a qual a testemunha foi considerada a dosagem de zero $\mathrm{g} \mathrm{m}^{-3}$ de fosfina, em atmosfera normal.

\section{RESULTADOS E DISCUSSÃO}

A determinação da eficácia do processo de fumigação no controle de todas as fases de desenvolvimento de $R$. dominica foi realizada por meio da contagem de insetos que se tornaram adultos.

\section{Avaliação da fase de ovo (2 a 6 dias de idade)}

Por ser um estádio imaturo, os ovos são considerados, assim como as pupas, mais tolerantes ao controle que os adultos, tanto em populações suscetíveis quanto em populações resistentes à fosfina. Apresentam-se os valores médios de eficácia (Tabela 1), obtidos em cada tratamento de atmosfera sintética contendo $0,25,0,50$ e $0,75 \mathrm{~g} \mathrm{~m}^{-3}$ de fosfina e atmosfera ambiente contendo $1,00 \mathrm{~g} \mathrm{~m}^{-3} \mathrm{de}$ fosfina, em cada período de exposição, na fase de ovo de $R$. dominica. No período de exposição de 24 horas, observa-se que, à medida que se aumentou a concentração de fosfina associada à atmosfera sintética, maior foi a eficácia nos ovos de $R$. dominica, embora em nenhum dos tratamentos tenha sido obtido um controle efetivo. $O$ tratamento em que se utilizou atmosfera ambiente e $1,00 \mathrm{~g} \mathrm{~m}^{-3}$ de fosfina, no período de exposição de 24 horas, não foi efetivo no controle de ovos e não diferiu significativamente do tratamento com atmosfera sintética e $0,75 \mathrm{~g} \mathrm{~m}^{-3} \mathrm{de}$ fosfina. Nos demais períodos de exposição, $72 \mathrm{e}$ 120 horas, os tratamentos com atmosfera sintética e atmosfera ambiente não diferiram entre si. Ressaltase que a eficácia de $100 \%$ no controle de ovos somente foi obtida nos tratamentos com atmosfera sin- 
tética e $0,25,0,50$ e $0,75 \mathrm{~g} \mathrm{~m}^{-3}$ de fosfina. Calderon \& Navarro (1980), realizando expurgos com concentrações de $\mathrm{CO}_{2}$ variando entre $5 \%$ e $30 \%$ e de $\mathrm{O}_{2}$ variando entre $2 \%$ e $8 \%$, obtiveram o porcentual máximo de $96 \%$ de mortalidade de ovos de $R$. dominica, em 96 horas de exposição, com temperatura em torno de $26^{\circ} \mathrm{C}$ e umidade relativa de $57 \%$. Desmarchelier \& Wohlgemuth (1984) obtiveram 50\% de mortalidade dos ovos de $R$. dominica em 7,9 horas, quando utilizaram dosagem elevada de fosfina associada a uma concentração de $25 \%$ de $\mathrm{CO}_{2}$, a $19^{\circ} \mathrm{C}$ e $70 \%$ de umidade relativa. Para uma mesma dosagem de fosfina e $75 \%$ de $\mathrm{CO}_{2}$, obteve-se $50 \%$ de mortalidade em 8,1 horas. Para os mesmos valores de temperatura e umidade relativa, o expurgo com dióxido de carbono na concentração de $75 \%$, o mesmo índice de mortalidade, $50 \%$, foi obtido em 94 horas.

\section{Avaliação da fase de larva do primeiro ínstar (8 a 13 dias de idade)}

Apresentam-se os valores médios de eficácia na fase de larva do primeiro ínstar na Tabela 2. Observa-se que, no período de exposição de 24 horas, a menor eficácia foi obtida no tratamento com atmosfera ambiente e $1,00 \mathrm{~g} \mathrm{~m}^{-3}$ de fosfina. Os tratamentos com atmosfera sintética e fosfina apresentaram tendência de melhor eficácia à medida que se aumentou a dose de fosfina. No entanto, no período de exposição de 72 horas, as maiores eficácias foram obtidas quando se utilizaram 0,25 e $0,75 \mathrm{~g} \mathrm{~m}^{-3}$ de fosfina e atmosfera sintética, e as menores, com atmosfera ambiente de $1,00 \mathrm{~g} \mathrm{~m}^{-3}$ de fosfina e atmosfera sintéti-

TABELA 1. Valores médios da eficácia (\% de mortalidade) dos tratamentos nas dosagens utilizadas, em cada período de exposição, na fase ovo de $R$. dominica ${ }^{1}$.

\begin{tabular}{llrr}
\hline Dose & \multicolumn{3}{c}{ Período de exposição (horas) } \\
\cline { 2 - 4 } & \multicolumn{1}{c}{24} & 72 & 120 \\
\hline$\left(0,25 \mathrm{~g} \mathrm{~m}^{-3}\right.$ de fosfina $)+\mathrm{CO}_{2}$ & $40,7 \mathrm{c}$ & $100,0 \mathrm{a}$ & $100,0 \mathrm{a}$ \\
$\left(0,50 \mathrm{~g} \mathrm{~m}^{-3} \mathrm{de}\right.$ fosfina $)+\mathrm{CO}_{2}$ & $51,8 \mathrm{~b}$ & $100,0 \mathrm{a}$ & $100,0 \mathrm{a}$ \\
$\left(0,75 \mathrm{~g} \mathrm{~m}^{-3}\right.$ de fosfina $)+\mathrm{CO}_{2}$ & $61,7 \mathrm{ab}$ & $100,0 \mathrm{a}$ & $100,0 \mathrm{a}$ \\
$1,00 \mathrm{~g} \mathrm{~m}^{-3}$ de fosfina & $65,0 \mathrm{a}$ & $95,6 \mathrm{a}$ & $99,3 \mathrm{a}$ \\
\hline & \multicolumn{3}{l}{} \\
1 & Médias seguidas da mesma letra nas colunas, em cada período de expo- \\
sição, não diferem entre si a 5\% de probabilidade pelo teste de Newman- \\
Keuls.
\end{tabular}

Pesq. agropec. bras., Brasília, v.35, n.6, p.1063-1069, jun. 2000 ca de $0,50 \mathrm{~g} \mathrm{~m}^{-3}$ de fosfina. $O$ controle efetivo de larvas do primeiro ínstar de $R$. dominica só foi alcançado quando o período de exposição foi de 120 horas. Ressalta-se, no entanto, que $100 \%$ de eficácia somente ocorreu quando se utilizaram $0,50 \mathrm{~g} \mathrm{~m}^{-3} \mathrm{de}$ fosfina em atmosfera sintética e $1,00 \mathrm{~g} \mathrm{~m}^{-3}$ de fosfina em atmosfera ambiente. Esses resultados confirmam o relato de Mueller (1994), isto é, a associação de atmosferas ricas em dióxido de carbono com baixas concentrações de fosfina, em temperaturas elevadas, são mais eficientes com menor período de exposição que a dose normalmente recomendada.

\section{Avaliação da fase de larva do segundo ínstar (14 a 18 dias de idade)}

A análise de variância da eficácia dos tratamentos com atmosfera sintética, contendo $0,25,0,50 \mathrm{e}$ $0,75 \mathrm{~g} \mathrm{~m}^{-3}$ de fosfina, e atmosfera ambiente com $1,00 \mathrm{~g} \mathrm{~m}^{-3}$ de fosfina, nos períodos de exposição de 24, 72 e 120 horas, mostrou, pelo teste F, a 5\% de probabilidade, que houve efeito significativo apenas do período de exposição sobre a mortalidade das larvas do segundo ínstar de $R$. dominica.

Na Fig. 1, observa-se o efeito do período de exposição sobre a eficácia na fase de larva do segundo ínstar, onde se verifica que a eficácia dos tratamentos com fosfina aumentou à medida que se aumentou o período de exposição. Price \& Mills (1988) enfatizam que o período de exposição é um fator mais crítico para fumigação com fosfina do que a dose utilizada do fumigante.

TABELA 2. Valores médios da eficácia ( $\%$ de mortalidade) dos tratamentos nas dosagens utilizadas, em cada período de exposição, na fase de larva do primeiro ínstar de R. dominica ${ }^{1}$.

\begin{tabular}{llcr}
\hline \multirow{2}{*}{ Dose } & \multicolumn{3}{c}{ Período de exposição (horas) } \\
\cline { 2 - 4 } & \multicolumn{1}{c}{24} & 72 & \multicolumn{1}{c}{120} \\
\hline$\left(0,25 \mathrm{~g} \mathrm{~m}^{-3}\right.$ de fosfina $)+\mathrm{CO}_{2}$ & $66,7 \mathrm{~b}$ & $96,7 \mathrm{a}$ & $96,3 \mathrm{a}$ \\
$\left(0,50 \mathrm{~g} \mathrm{~m}^{-3}\right.$ de fosfina $)+\mathrm{CO}_{2}$ & $78,2 \mathrm{ab}$ & $73,7 \mathrm{~b}$ & $100,0 \mathrm{a}$ \\
$\left(0,75 \mathrm{~g} \mathrm{~m}^{-3}\right.$ de fosfina $)+\mathrm{CO}_{2}$ & $94,2 \mathrm{a}$ & $93,0 \mathrm{a}$ & $99,3 \mathrm{a}$ \\
$1,00 \mathrm{~g} \mathrm{~m}^{-3}$ de fosfina & $44,7 \mathrm{c}$ & $63,8 \mathrm{~b}$ & $100,0 \mathrm{a}$ \\
\hline
\end{tabular}

${ }^{1}$ Médias seguidas da mesma letra nas colunas, em cada período de exposição, não diferem entre si a $5 \%$ de probabilidade pelo teste de NewmanKeuls 


\section{Avaliação da fase de larva do terceiro ínstar (19 a 23 dias de idade)}

Na Tabela 3, apresentam-se os valores médios da eficácia na fase de larva do terceiro ínstar de $R$. dominica. No período de exposição de 24 horas, o tratamento com atmosfera sintética e $0,75 \mathrm{~g} \mathrm{~m}^{-3} \mathrm{de}$ fosfina apresentou maior eficácia que os demais tratamentos. No período de exposição de 72 horas, todos os tratamentos com atmosfera sintética contendo fosfina foram mais eficazes que o tratamento com fosfina em atmosfera ambiente. Todos os tratamentos foram eficazes sobre larvas do terceiro ínstar do $R$. dominica quando o período de exposição foi de 120 horas. Esses resultados coincidem com os obtidos para larvas do primeiro e segundo ínstares (Tabela 1 e Fig. 1).

\section{Avaliação da fase de larva do quarto ínstar (24 a 28 dias de idade)}

Por ser a última fase do estádio larval, antes de empupar há uma redução na atividade respiratória do inseto, tornando este estádio menos suscetível ao controle, em comparação com as larvas dos ínstares anteriores. Na Tabela 4, apresentam-se os

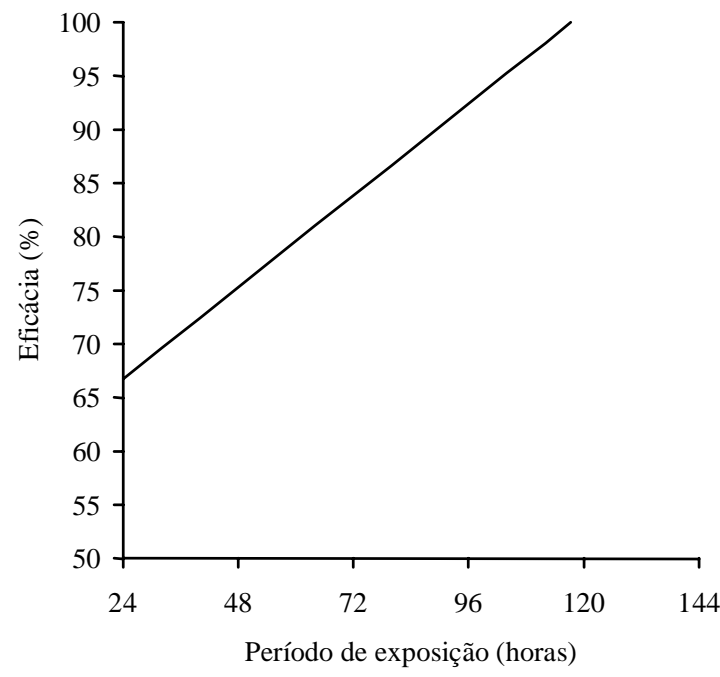

FIG. 1. Estimativa da eficácia dos tratamentos nas larvas do segundo ínstar de $R$. dominica, de acordo com o período de exposição $\left(y=58,2158+0,3561 x ; R^{2}=0,92\right)$ valores médios da eficácia na fase de larva do quarto ínstar de $R$. dominica. No período de exposição de 24 horas, verifica-se que, à medida que se aumentou a concentração de fosfina na presença de atmosfera sintética, maior foi a eficácia. Observa-se, também, que os tratamentos com atmosfera sintética e 0,50 e $0,75 \mathrm{~g} \mathrm{~m}^{-3}$ de fosfina não diferiram significativamente do tratamento com $1,00 \mathrm{~g} \mathrm{~m}^{-3}$ de fosfina na atmosfera ambiente. No entanto, no período de exposição de 72 horas, os tratamentos com atmosfera sintética e fosfina foram mais eficazes sobre as larvas de quarto ínstar do que o tratamento com atmosfera ambiente e $1,00 \mathrm{~g} \mathrm{~m}^{-3}$ de fosfina. No período de exposição de 120 horas, a menor eficácia ocorreu quando se utilizou $0,25 \mathrm{~g} \mathrm{~m}^{-3}$ de fosfina na atmosfera sintética. Os demais tratamentos não diferiram significativamente entre si.

Em geral, insetos na fase de larva do quarto ínstar de $R$. dominica foram mais resistentes em comparação com os demais. Gonçalves (1997), realizando expurgo com dióxido de carbono em concentrações de $30 \%$ a $60 \%$ e $\mathrm{N}$ em $40 \%$ a $70 \%$, à temperatura média de $20^{\circ} \mathrm{C}$, em 5,10 e 15 dias de exposição, concluiu que as larvas do quarto ínstar, seguidas pelas pupas, são as fases mais resistentes do desenvolvimento do $R$. dominica.

\section{Avaliação da fase de pupa (idade entre 29 e 32 dias)}

Na Tabela 5, apresentam-se os valores médios da eficácia dos tratamentos na fase de pupa de

TABELA 3. Valores médios da eficácia (\% de mortalidade) dos tratamentos nas dosagens utilizadas, em cada período de exposição, na fase de larva do terceiro ínstar de R. dominica ${ }^{1}$.

\begin{tabular}{lccc}
\hline Dose & \multicolumn{3}{c}{ Período de exposição (horas) } \\
\cline { 2 - 4 } & 24 & 72 & 120 \\
\hline$\left(0,25 \mathrm{~g} \mathrm{~m}^{-3}\right.$ de fosfina $)+\mathrm{CO}_{2}$ & $66,5 \mathrm{~b}$ & $90,7 \mathrm{a}$ & $100,0 \mathrm{a}$ \\
$\left(0,50 \mathrm{~g} \mathrm{~m}^{-3}\right.$ de fosfina $)+\mathrm{CO}_{2}$ & $74,4 \mathrm{~b}$ & $90,2 \mathrm{a}$ & $100,0 \mathrm{a}$ \\
$\left(0,75 \mathrm{~g} \mathrm{~m}^{-3}\right.$ de fosfina $)+\mathrm{CO}_{2}$ & $91,8 \mathrm{a}$ & $95,7 \mathrm{a}$ & $98,6 \mathrm{a}$ \\
$1,00 \mathrm{~g} \mathrm{~m}^{-3}$ de fosfina & $65,5 \mathrm{~b}$ & $68,0 \mathrm{~b}$ & $98,3 \mathrm{a}$ \\
\hline
\end{tabular}

${ }^{1}$ Médias seguidas da mesma letra nas colunas, em cada período de exposição, não diferem entre si a $5 \%$ de probabilidade pelo teste de NewmanKeuls. 
R. dominica. No período de exposição de 24 horas, observa-se que os tratamentos com atmosfera sintética e fosfina foram mais eficazes, principalmente quando se aumentou a concentração de fosfina de 0,25 para 0,50 e $0,75 \mathrm{~g} \mathrm{~m}^{-3}$, que o tratamento com atmosfera ambiente e $1,00 \mathrm{~g} \mathrm{~m}^{-3}$ de fosfina. Nos períodos de exposição de 72 e 120 horas, o mesmo comportamento foi observado, ou seja, à medida que se aumentou a concentração de fosfina na presença da atmosfera sintética, maior foi a mortalidade das pupas. A maior eficácia alcançada foi nos tratamentos com atmosfera sintética e 0,50 e $0,75 \mathrm{~g} \mathrm{~m}^{-3}$ de fosfina, nos períodos de exposição de 72 e 120 horas. No mesmo período de exposição, todos os tratamentos com atmosfera sintética e fosfina foram mais eficazes que o tratamento com atmosfera ambiente, o que coinci-

TABELA 4. Valores médios da eficácia (\% de mortalidade) dos tratamentos nas dosagens utilizadas, em cada período de exposição, na fase de larva do quarto ínstar de R. dominica ${ }^{1}$

\begin{tabular}{llcr}
\hline Dose & \multicolumn{3}{c}{ Período de exposição (horas) } \\
\cline { 2 - 4 } & \multicolumn{1}{c}{24} & 72 & 120 \\
\hline$\left(0,25 \mathrm{~g} \mathrm{~m}^{-3}\right.$ de fosfina $)+\mathrm{CO}_{2}$ & $53,6 \mathrm{~b}$ & $78,9 \mathrm{a}$ & $79,1 \mathrm{~b}$ \\
$\left(0,50 \mathrm{~g} \mathrm{~m}^{-3}\right.$ de fosfina $)+\mathrm{CO}_{2}$ & $68,4 \mathrm{ab}$ & $84,8 \mathrm{a}$ & $100,0 \mathrm{a}$ \\
$\left(0,75 \mathrm{~g} \mathrm{~m}^{-3}\right.$ de fosfina $)+\mathrm{CO}_{2}$ & $77,4 \mathrm{a}$ & $93,5 \mathrm{a}$ & $96,7 \mathrm{a}$ \\
$1,00 \mathrm{~g} \mathrm{~m}^{-3}$ de fosfina & $65,9 \mathrm{ab}$ & $55,4 \mathrm{~b}$ & $98,1 \mathrm{a}$ \\
\hline
\end{tabular}

${ }^{1}$ Médias seguidas da mesma letra nas colunas, em cada período de exposição, não diferem entre si a $5 \%$ de probabilidade pelo teste de NewmanKeuls

TABELA 5. Valores médios da eficácia (\% de mortalidade) dos tratamentos nas dosagens utilizadas, em cada período de exposição, na fase de pupa de $R$. dominica ${ }^{1}$.

\begin{tabular}{lccc}
\hline Dose & \multicolumn{3}{c}{ Período de exposição (horas) } \\
\cline { 2 - 4 } & 24 & 72 & 120 \\
\hline$\left(0,25 \mathrm{~g} \mathrm{~m}^{-3}\right.$ de fosfina $)+\mathrm{CO}_{2}$ & $44,7 \mathrm{~b}$ & $64,5 \mathrm{~b}$ & $70,9 \mathrm{~b}$ \\
$\left(0,50 \mathrm{~g} \mathrm{~m}^{-3}\right.$ de fosfina $)+\mathrm{CO}_{2}$ & $65,4 \mathrm{a}$ & $96,7 \mathrm{a}$ & $96,5 \mathrm{a}$ \\
$\left(0,75 \mathrm{~g} \mathrm{~m}^{-3}\right.$ de fosfina $)+\mathrm{CO}_{2}$ & $72,9 \mathrm{a}$ & $99,2 \mathrm{a}$ & $99,3 \mathrm{a}$ \\
$1,00 \mathrm{~g} \mathrm{~m}^{-3}$ de fosfina & $28,8 \mathrm{~b}$ & $42,0 \mathrm{c}$ & $70,1 \mathrm{~b}$ \\
\hline
\end{tabular}

1 Médias seguidas da mesma letra nas colunas, em cada período de exposição, não diferem entre si a $5 \%$ de probabilidade pelo teste de NewmanKeuls de com os resultados obtidos em todas as fases larvais (Tabelas 2, 3 e 4 e Fig. 1). Segundo Price \& Mills (1988), estádios imaturos, principalmente ovos e pupas, são mais tolerantes que os adultos, tanto em populações suscetíveis quanto em populações resistentes à fosfina. A resistência ao dióxido de carbono é também maior em estádios imaturos do que em adultos em várias espécies de insetos de grãos armazenados (Bell \& Armitage, 1980).

\section{Avaliação da fase adulta (acima de 32 dias de idade)}

Apresenta-se, na Tabela 6, os valores médios da eficácia dos tratamentos sobre os insetos adultos de $R$. dominica. Nos períodos de exposição de 24 e 72 horas, observa-se que todos os tratamentos com atmosfera sintética e fosfina foram mais eficazes que os tratamentos com atmosfera ambiente e $1,00 \mathrm{~g} \mathrm{~m}^{-3}$ de fosfina. Verifica-se que no período de exposição de 72 horas, a menor dose de fosfina $\left(0,25 \mathrm{~g} \mathrm{~m}^{-3}\right)$, associada à atmosfera sintética, promoveu $100 \%$ de eficácia no controle dos insetos adultos. Esses resultados coincidem com os obtidos por Desmarchelier \& Wohlgemuth (1984), ou seja, o controle na fase adulta foi obtido em um tempo menor em relação às outras fases de $R$. dominica . Calil (1995), trabalhando com populações resistentes e suscetíveis de $R$. dominica à fosfina, verificou que a fase adulta é uma das fases mais suscetíveis dessa espécie. O mesmo comportamento foi observado por Gonçalves (1997), em trabalho realizado com a mistura de dióxido de carbono e nitrogênio.

TABELA 6. Valores médios da eficácia (\% de mortalidade) dos tratamentos nas dosagens utilizadas, em cada período de exposição, na fase adulta de $R$. dominica ${ }^{1}$.

\begin{tabular}{lrrr}
\hline Dose & \multicolumn{3}{c}{ Período de exposição (horas) } \\
\cline { 2 - 4 } & \multicolumn{1}{c}{24} & 72 & 120 \\
\hline$\left(0,25 \mathrm{~g} \mathrm{~m}^{-3}\right.$ de fosfina $)+\mathrm{CO}_{2}$ & $79,3 \mathrm{~b}$ & $100,0 \mathrm{a}$ & $100,0 \mathrm{a}$ \\
$\left(0,50 \mathrm{~g} \mathrm{~m}^{-3}\right.$ de fosfina $)+\mathrm{CO}_{2}$ & $93,9 \mathrm{a}$ & $98,6 \mathrm{a}$ & $100,0 \mathrm{a}$ \\
$\left(0,75 \mathrm{~g} \mathrm{~m}^{-3}\right.$ de fosfina $)+\mathrm{CO}_{2}$ & $100,0 \mathrm{a}$ & $100,0 \mathrm{a}$ & $100,0 \mathrm{a}$ \\
$1,00 \mathrm{~g} \mathrm{~m}^{-3}$ de fosfina & $66,3 \mathrm{c}$ & $78,7 \mathrm{~b}$ & $98,9 \mathrm{a}$ \\
\hline
\end{tabular}

${ }^{1}$ Médias seguidas da mesma letra nas colunas, em cada período de exposição, não diferem entre si a $5 \%$ de probabilidade pelo teste de NewmanKeuls. 


\section{CONCLUSÕES}

1. O aumento do período de exposição resulta no aumento da eficácia dos tratamentos com atmosfera sintética $\left(21 \%\right.$ de $\left.\mathrm{CO}_{2}\right)$ e atmosfera ambiente associados à fosfina, em todas as fases de desenvolvimento do inseto $R$. dominica.

2. O período de exposição de 24 horas só é eficaz no tratamento de insetos adultos com atmosfera sintética e $0,75 \mathrm{~g} \mathrm{~m}^{-3}$ de fosfina.

3. Todos os tratamentos com atmosfera sintética associada à fosfina controlam eficazmente ovos de R. dominica a partir de 72 horas de exposição.

4. O controle efetivo ( $100 \%$ de eficácia) de todas as fases de desenvolvimento do inseto $R$. dominica é obtido, em geral, com atmosfera sintética associada a 0,50 e $0,75 \mathrm{~g} \mathrm{~m}^{-3}$ de fosfina, no período de exposição de 120 horas.

5. A concentração mínima de fosfina associada à atmosfera sintética requerida para o controle efetivo de todas as fases de $R$. dominica é de $0,50 \mathrm{~g} \mathrm{~m}^{-3}$ no período de exposição de 120 horas.

6. Ovos e adultos de $R$. dominica são as fases mais suscetíveis, e pupa, a mais resistente.

\section{REFERÊNCIAS}

BELL, C.H.; ARMITAGE, D.M. Alternative storage practices. In: SAUER, D.B. (Ed.). Storage of cereal grains and their products. 4.ed. Saint Paul : American Association of Cereal Chemists, 1980 p.249-274

CALDERON, M.; NAVARRO, S. Synergistic effect of $\mathrm{CO}_{2}$ and $\mathrm{O}_{2}$ mixtures on two stored grain insect pests. In: SHEJBAL, J. (Ed.). Controlled atmosphere storage of grains, Amsterdam Elsevier, 1980. p.101-118.

CALIL, A.C.P. Efeito de doses de fosfina e períodos de exposição, na mortalidade de formas adulta $e$ imaturas de Rhyzopertha dominica (Coleoptera: Bostrichidae), em trigo. Viçosa : UFV, 1995. 67p. Tese de Doutorado.

COMPÊNDIO de defensivos agrícolas; guia prático de produtos fitossanitários para uso agrícola. 5.ed. São Paulo : Organização Andrei, 1996.250p.

DESMARCHELIER, J.M.; WOHLGEMUTH, R Response of several species of insects to mixtures of phosphine and carbon dioxide. In: RIPP, B.E.; BANKS, H.J.; BOND, E.J.; CALVERLEY,D.J.; JAY, E.G.; NAVARRO, S. (Ed.). Controlled atmosphere and fumigation in grain storages. Perth : ACIAR, 1984. p.237-245.

GALLO, D.; NAKANO, O.; SILVEIRANETO, S.; CARVALHO, R.P.L.; BATISTA, G.C.; BERTI FILHO, E.; PARRA, J.R.P.; ZUCCHI, R.A.; ALVES, S.B.; VENDRAMIN, J.D. Manual de entomologia agrícola. São Paulo : Agronômica Ceres, 1988. 649p.

GONÇALVES, R.A. Preservação da qualidade tecnológica de trigo (Triticum aestivum $\mathrm{L}$.) e controle da Rhyzopertha dominica (F.) durante o armazenamento em atmosfera controlada com $\mathbf{C O}_{2}$ e $\mathbf{N}_{2}$. Lavras : UFLA, 1997. 52p. Tese de Mestrado.

LEONG, E.C.W.; HO, S.H. Response of Liposcelis bostrychophila and L. entomophila (Psocoptera) to carbon dioxide. In: INTERNATIONAL WORKING CONFERENCE ON STORED PRODUCT PROTECTION, 6., 1994, Canbera. Proceedings. Canbera : [s.n.], 1994. p.108-112.

MUELLER, D.K. A new method of using low levels of phosphine in combination with heat and carbon dioxide. In: INTERNATIONAL WORKING CONFERENCE ON STORED PRODUCT PROTECTION, 6., 1994, Canbera. Proceedings. Canbera : [s.n.], 1994. p.123-125.

PACHECO, I.A.; SARTORI, M.R.; BOLONHEZI, S. Resistance to malathion, pirimiphos-methyl and feinitrothion in Coleoptera from stored grains. In: INTERNATIONAL WORKING CONFERENCE ON STORED-PRODUCT PROTECTION, 5. 1990, Bordeaux. Proceedings. Bordeaux : INRAStation de Physiologie Végétable, 1990. p. 1029-1037.

PRICE, L.A.; MILLS, K.A. The toxicity of phosphine to the immature stages of resistant and susceptible strains of some common stored product beetles and implications for their control. Journal of Stored Products Research, Oxford, n.24, p.51-59, 1988.

SARTORI, M.R.; PACHECO, I.A.; VILAR, R.M.G. Resistance to phosphine in stored grain insects in Brazil. II. In: INTERNATIONAL WORKING CONFERENCE ON STORED PRODUCT PROTECTION, 5., 1990, Bordeaux. Proceedings. Bordeaux : INRA-Station de Physiologie Végétable, 1990. p.1041-1049.

Pesq. agropec. bras., Brasília, v.35, n.6, p.1063-1069, jun. 2000 